\title{
Transition region oscillations above sunspots
}

\begin{abstract}
A. Fludra
Space Science \& Technology Department, Rutherford Appleton Laboratory, Chilton, Didcot, Oxfordshire, OX11 0QX, UK

e-mail: A.Fludra@rl.ac.uk

Received 8 September 2000 / Accepted 22 December 2000

Abstract. We present observations of three sunspots made in the EUV wavelength range with the Coronal Diagnostic Spectrometer (CDS) on SOHO. We examine time series of line intensities obtained with a 15-s cadence and calculate their Fourier power spectra and the wavelet transforms. We find oscillations in the chromosphere and transition region above sunspots in the temperature range $210^{4}-410^{5} \mathrm{~K}$. Most of the spectral power is contained in the $5-8 \mathrm{mHz}$ frequency range, and the dominant frequency is $5.9 \mathrm{mHz}$ (170 s period). We find a relationship between the oscillations and sunspot plumes which are compact features located above sunspots and bright in the transition region lines emitted between $1.710^{5}$ and $410^{5} \mathrm{~K}$. When the CDS slit crosses a sunspot plume, the bright O IV $554.5 \AA$ and O V $629.7 \AA$ lines show clear 3 min oscillations. An extreme alternative interpretation that excludes the presence of oscillations in sunspot plumes carries the requirement that the adjacent low-intensity plasma oscillate with high relative amplitudes greater than 50 percent. We also observe oscillations in a fainter area above a sunspot, outside the sunspot plume. The oscillations seen at different temperatures are compared. Using the wavelet transform, we find variability of the oscillation period in the range 120-200 s.
\end{abstract}

Key words. Sun: transition region - Sun: oscillations - Sun: UV radiation - sunspots

\section{Introduction}

Oscillations in the chromosphere above sunspots have been studied since the late 1960's (see a review by Lites 1992). First observations of sunspot oscillations at higher temperatures characteristic of the transition region between the chromosphere and corona became possible using UV observations from the UVSP instrument on the Solar Maximum Mission (e.g., Gurman et al. 1982). They observed oscillations of the C IV $1548.19 \AA$ line, emitted at temperatures close to $10^{5} \mathrm{~K}$. Oscillations were seen mostly in velocity but four out of twelve time series analysed by Gurman et al. (1982) also had significant power in intensity oscillations. Further results on the transition region oscillations above sunspots, based on data from the Coronal Diagnostic Spectrometer (CDS - Harrison et al. 1995) and the SUMER instrument on the Solar and Heliospheric Observatory (SOHO), are given by Fludra (1999a,b) and Brynildsen (1999a, 2000). CDS data were used to study oscillations in intensity, while SUMER measured oscillations both in velocity and intensity.

Some observational characteristics of sunspot oscillations have been derived previously. The transition region oscillations are seen in the temperature range up to $410^{5} \mathrm{~K}$. The dominant period is three minutes, but several peaks with frequencies between 5 and $8 \mathrm{mHz}$ are also

Send offprint requests to: A. Fludra,

e-mail: A.Fludra@rl.ac.uk present in the Fourier power spectra. Gurman \& Leibacher (1984) suggested that upward propagating acoustic waves are responsible for these oscillations. This has been confirmed by Brynildsen et al. (1999a), with an indication that the waves can be nonlinear in some sunspots.

There remain several issues that have not been fully addressed yet and that can be resolved through special observing sequences and application of new analysis methods:

(1) The structure of the power spectrum and the variability of the oscillation period need to be investigated in more detail, and observations with a long time series are needed to obtain good frequency resolution. Lites (1986) suggested that separate peaks in the Fourier power spectrum of chromospheric oscillations are cospatial oscillatory modes, rather than a result of averaging oscillatory modes in separate flux tubes and that the distribution of power varies from point to point within the umbra. This question needs to be investigated for the transition region oscillations.

(2) Chromospheric oscillations are seen in all areas of the umbra (e.g., Lites et al. 1982). Maltby et al. (1999) also find that the entire umbral transition region oscillates. However, the transition region above a sunspot can be highly non-uniform, particularly at temperatures $1.5-410^{5} \mathrm{~K}$, where one can see so called sunspot plumes, i.e., prominent features in the transition region above sunspots (Foukal et al. 1974; Foukal 1976), first seen in the 
CDS data as very bright features in the O V $629.7 \AA$ line (peak formation at $2.210^{5} \mathrm{~K}$ ) by Fludra et al. (1997) and described in detail by Brynildsen et al. (1999b). Sunspot plumes do not cover the whole umbra nor are they necessarily located entirely above the umbra. Fludra (1999a,b) found 3 min oscillations in intensity of transition region lines, spatially connected to bright emission of sunspot plumes to within the resolution of the CDS instrument. On the other hand, Brynildsen et al. (2000) presented data with $1^{\prime \prime}$ spatial resolution suggesting that the maximum oscillation power is not co-spatial with sunspot plumes. Thus, the spatial location of the transition region oscillations at different temperatures and the possibility of oscillations taking place in sunspot plumes need clarification.

(3) The temperature dependence of the oscillations and the relationship between the chromospheric and transition region oscillations need further characterization.

In this paper we describe observations of oscillations in one chromospheric line (formed at $210^{4} \mathrm{~K}$ ) and several transition region lines emitted in the temperature range $910^{4}-410^{5} \mathrm{~K}$ made with CDS. We present several time series, one to four hours long, and obtain Fourier power spectra with high resolution in frequency. We also apply a wavelet transform to obtain information on time variability of the oscillation period, and we show that power spectra vary from sunspot to sunspot, and that in any particular sunspot the oscillation period can vary in a range 120-200 s. We typically find a spatial correlation of the oscillations with the strong intensity enhancements in sunspot plumes, however, oscillations can also take place in areas of low intensity. Unique features of our analysis are: long time series giving good frequency resolution of the power spectrum, an application of both Fourier and wavelet analysis, and observations made both with a fixed pointing and with solar rotation tracking.

Instrumental details relevant to the observations are given in Sect. 2. Results for each sunspot are given in Sect. 3 and the discussion and summary are in Sect. 4 .

\section{Instrument and the method}

The SOHO Coronal Diagnostic Spectrometer (CDS; Harrison et al. 1995) consists of a Wolter-Schwarzschild type II grazing incidence telescope focused at the common entrance slit of a pair of spectrometers: normal incidence (NIS) and grazing incidence (GIS). The data discussed in this paper were recorded by the normal incidence spectrometer using a $4 \times 240$ arcsec slit to obtain a time series, and a $2 \times 240 \operatorname{arcsec}$ slit to produce a rastered image that provides context information. Typically, a time series had a $10 \mathrm{~s}$ exposure time and $5.6 \mathrm{~s}$ telemetry overhead, giving a $15.6 \mathrm{~s}$ cadence for several hours.

The data are processed using standard CDS calibration software which removes cosmic-ray strikes and corrects the CDS data for the CCD read-out bias, the flatfield image, and the detector "burn-in" due to the repeated exposure of strong lines. Counts/pixel are converted to photon-events/pixel/s. The total intensity in each
Table 1. EUV lines used in sunspot observations

\begin{tabular}{lll}
\hline Ion & $\begin{array}{l}\text { Wavelength } \\
(\AA)\end{array}$ & $\begin{array}{l}\text { Temperature }^{a} \\
(\mathrm{~K})\end{array}$ \\
\hline $\mathrm{He} \mathrm{I}$ & 584.33 & $2.010^{4}$ \\
O III & 599.6 & $1.010^{5}$ \\
O IV & 554.5 & $1.710^{5}$ \\
O V & 629.7 & $2.210^{5}$ \\
$\mathrm{Ne} \mathrm{IV}$ & 543.89 & $1.710^{5}$ \\
$\mathrm{Ne} \mathrm{V}$ & 572.33 & $2.810^{5}$ \\
$\mathrm{Ne} \mathrm{VI}$ & 562.8 & $4.010^{5}$ \\
$\mathrm{Mg} \mathrm{IX}$ & 368.06 & $9.510^{5}$ \\
\hline
\end{tabular}

a Temperature of the peak emissivity.

spectral line is calculated by summing the whole line profile and subtracting the instrumental background.

The observations were made in two modes: fixed pointing and tracking. Fixed pointing was used more often because it allows one to obtain a full scan of the sunspot and the surrounding area. The solar tracking requires a precise selection of the target which is more difficult given a $5^{\prime \prime}$ uncertainty in the CDS pointing and a small oscillating area. Each time series was preceded or followed by a rastered image which helps place the time series in the context of the image of the surrounding area. The fixed-pointing time series always had the same pointing coordinates as the raster, therefore the CDS pointing mechanism did not move between the raster and the time series, and there is no uncertainty in the relative position of the time series with respect to the raster and to any particular features seen in the chromosphere or the transition region. The tracking time series has the initial pointing the same as the raster and then advances in 2 or 4 arcsec steps using a very precise mechanism that rotates the scanning mirror. Thus, the tracking time series can also be precisely related to the rastered image.

Overlays of the CDS raster with data from other instruments, for example, with the white light sunspot images from the MDI instrument on SOHO, are subject to the $5^{\prime \prime}$ pointing uncertainty of the CDS. However, it is usually possible to spatially correlate the CDS rastered images in the chromospheric line of He I $584 \AA$ with an image in the He II 304 filter from the EIT instrument and reduce the pointing uncertainty to below $2^{\prime \prime}$.

Spectral lines present in the time series analysed in this paper are listed in Table 1. A summary of observations is given in Table 2.

\section{Results}

\subsection{NOAA 8369, 30 Oct. 1998}

The sunspot in NOAA active region 8369 was observed with CDS on 30 October 1998. The time series was 
Table 2. CDS observations of sunspot oscillations ${ }^{a}$

\begin{tabular}{lllllll}
\hline NOAA & CDS Date & CDS Time $^{b}$ & EIT Time & MDI Time & Tracking & CDS Lines \\
\hline 8369 & 30 Oct. 1998 & $08: 08: 48$ & $07: 19: 43$ & $08: 05: 34$ & OFF & Ne IV, Ne V, Ne VI, O V, Mg IX \\
8504 & 03 Apr. 1999 & $08: 45: 30$ & $07: 25: 53$ & $04: 47: 33$ & ON & He I, O IV, O V, Ne VI, Mg IX \\
8552 & 30 May 1999 & $13: 47: 46$ & $13: 18: 00$ & $11: 11: 33$ & OFF & He I, O III, O IV, O V, Ne VI \\
8552 & 31 May 1999 & $18: 34: 30$ & missing & not used & ON & He I, O IV, O V, Ne VI, Mg IX \\
\hline
\end{tabular}

a All time series taken using a $4^{\prime \prime} \times 240^{\prime \prime}$ slit.

${ }^{b}$ Start time of the CDS time series.

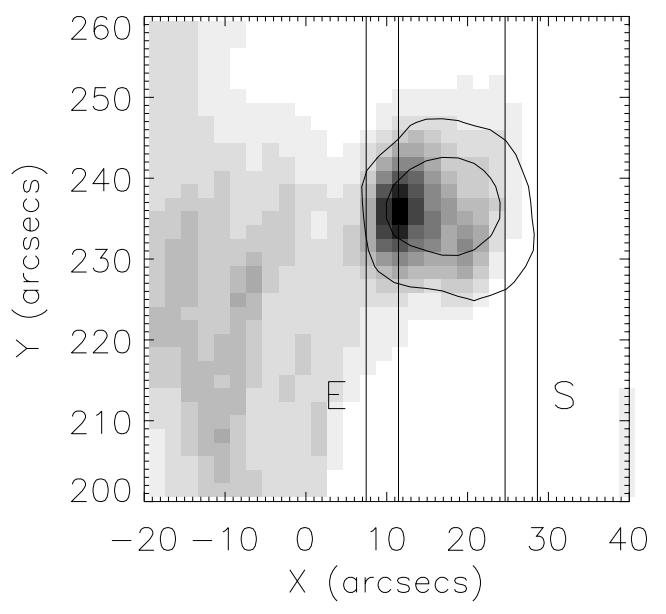

Fig. 1. A part of the CDS raster image of NOAA active region 8369 in the O V $629.7 \AA$ line on 30 October 1998. The colour table is reversed. The dark feature is a sunspot plume. The vertical lines show the CDS slit locations at the start ("S") and at the end ("E") of observation. The contours of the sunspot's umbra and penumbra are indicated

obtained using a $4^{\prime \prime} \times 240^{\prime \prime}$ slit and a 15 -s cadence $(10 \mathrm{~s}$ exposure time plus $5 \mathrm{~s}$ telemetry overhead). The slit was pointed to the right-hand side of the sunspot and kept at a fixed pointing while the sunspot moved with the solar rotation. The following lines in the normal incidence range were recorded: Ne IV $543.9 \AA$, Ne V 572.3, Ne VI 562.8, O V 629.7, and Mg IX $368.06 \AA$.

The time series was preceded and followed by a $2^{\prime} \times 2^{\prime}$ raster made with the $2^{\prime \prime} \times 240^{\prime \prime}$ slit in 15 spectral lines which give images of the area surrounding the sunspot in different temperatures. Figure 1 shows a $60^{\prime \prime} \times 60^{\prime \prime}$ area extracted from the CDS raster in the O V $629.7 \AA$ line. The feature with the largest intensity (shown dark in Fig. 1) is a sunspot plume. The vertical lines denote the location of the CDS slit at the start and at the end of the analysed time series. The positions of the CDS slit in Fig. 1 have been adjusted for solar rotation. At the time of observation the spacecraft was rolled so that the solar North is rotated by 9.5 degrees anticlockwise with respect to the vertical. Therefore, the solar features moved at an 80.5 degree angle to the CDS slit. To obtain more precise pointing coordinates, the pointing of the CDS raster has been adjusted by spatially correlating the CDS raster in

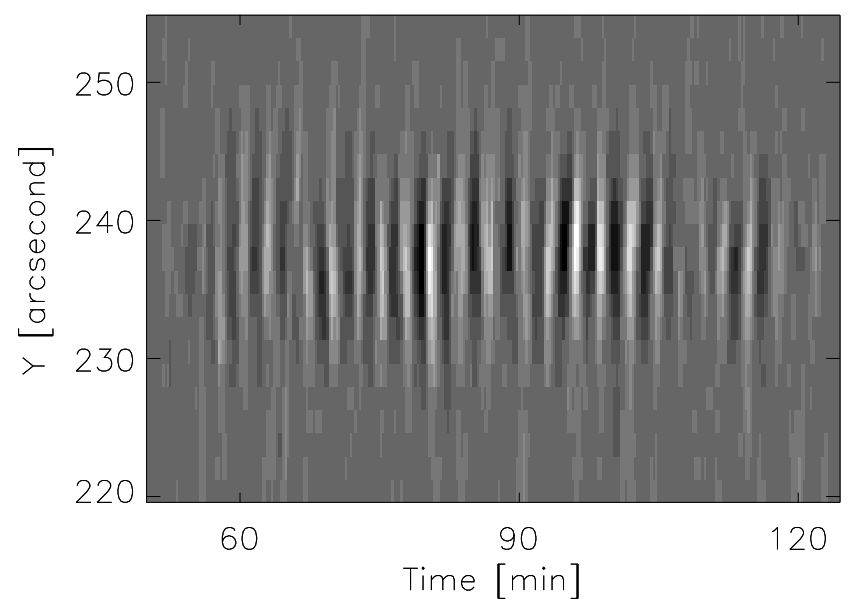

Fig. 2. Time variability of the intensity of the O V $629.7 \AA$ line along the CDS slit, during the observation of NOAA 8369 on 30 October 1998. Time series starts at 08:08:48 UT. The scale along the $Y$ axis is arcsec. One pixel is $1.68^{\prime \prime}$. The trend has been subtracted from each pixel's time history

the He I $584 \AA$ line with the He II $304 \AA$ image recorded by the SOHO/EIT instrument at 07:19:43 UT. The contours in Fig. 1 denote the sunspot's umbra and penumbra, and have been derived from a white light image observed by the MDI instrument on SOHO at 08:05:34 UT.

The sunspot plume was seen in all transition region lines recorded in the time series. However, oscillations were prominent only in the O V $629.7 \AA$ line. The oscillations are detectable in the Ne VI line, but the time series is rather noisy and the oscillation amplitude is small. Almost no oscillations were seen in the Ne IV and Ne V lines. Therefore, we focus the analysis on the O V $629.7 \AA$ line only.

Figure 2 shows the time variability of the $\mathrm{O} \mathrm{V}$ line intensity along a portion of the slit after subtracting the trend, where the trend for each spatial pixel is calculated as a 30-point running average of the intensity series. White colour denotes the peaks, black denotes the troughs of the oscillations.

Figure $3 \mathrm{a}$ shows the time history of the $\mathrm{O} \mathrm{V}$ line intensity, summed over 10 arcsec ( 6 pixels) along the slit which includes the brightest part of the sunspot plume. Figure $3 \mathrm{~b}$ shows the quantity (intensity/trend -1 ). 

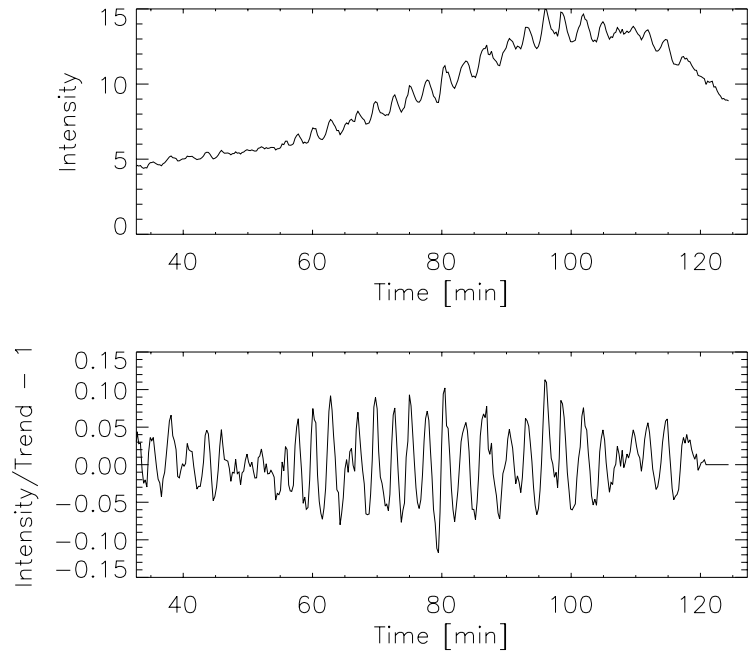

Fig. 3. a) Top panel: time history of the $\mathrm{O} V 629.7 \AA$ line intensity integrated over the sunspot plume area $\left(10^{\prime \prime}\right.$ along the slit) in NOAA active region 8369 on 30 October 1998. b) Bottom panel: relative O V intensity calculated as (intensity/trend -1). The slit was kept at a fixed pointing during the observation

The fast Fourier transform was applied to the time series from Fig. 3b. The Fourier power spectrum is shown in Fig. 4. There are two power peaks at the frequency of $5.5 \mathrm{mHz}(180 \mathrm{~s}$ period) and $6.3 \mathrm{mHz}(160 \mathrm{~s}$ period). The presence of two peaks in the power spectrum could arise for a number of reasons. First, it could represent a simultaneous presence of two oscillation periods. It is well known that if two sine signals of equal amplitude and close periods $P_{1}$ and $P_{2}$ are added, the amplitude of the resulting signal is modulated with a "beat" period of $1 / P_{b}=\left|1 / P_{1}-1 / P_{2}\right|$. For example, if $P_{1}=160 \mathrm{~s}$ and $P_{2}=180 \mathrm{~s}$, the envelope of the signal would reach zero every $1440 \mathrm{~s}$. Since this modulation pattern is not seen in the observed time series, a simultaneous presence of two oscillation frequencies seems unlikely. Second, two power peaks could also mean that the plasma in the field of view oscillated with one period for part of the time, and then the period changed to a different value for the remainder of the observation. A third possibility is an oscillation with one period of around $170 \mathrm{~s}$, where the amplitude of the time series is modulated by another periodic function with much longer period, in which case the Fourier transform would also produce two close peaks in the power spectrum.

If the oscillation frequency changes in time, the Fourier analysis done on the whole time series cannot provide the full information about when the frequency change occurred and whether it was gradual or step-like. Clearly, another method is needed to help us investigate this possibility.

Lites (1986) investigated variability of periods of umbral chromospheric oscillations using Fourier spectra of parts of the time series. He calculated the power spectra for restricted regions of the entire time series, by applying a 30 min mask and displacing it along the time series

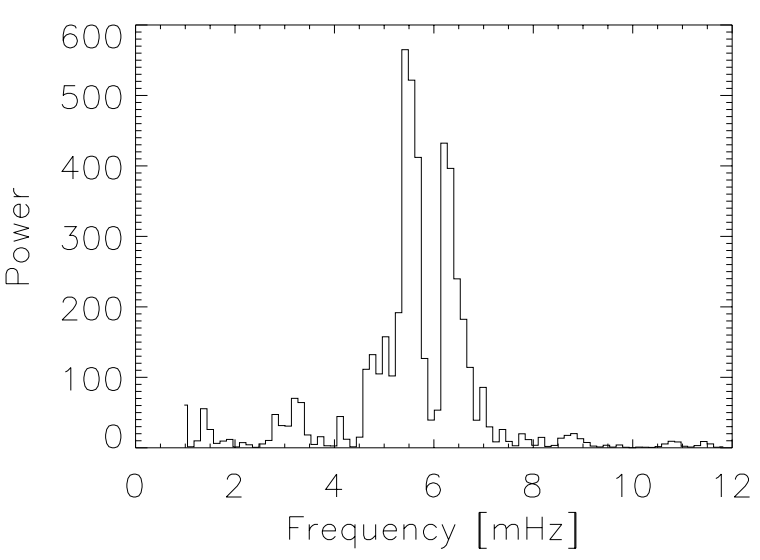

Fig. 4. Fourier power spectrum of the sunspot oscillations in the O V $629.7 \AA$ line intensity in NOAA active region 8369 on 30 October 1998. The line intensity has been summed over the six brightest pixels $\left(10^{\prime \prime}\right)$ along the slit

with a 200-s step. Figure 2 in Lites (1986) shows how the peak of the power spectrum changes with time, moving in frequency between 5 and $7 \mathrm{mHz}$. Lites concluded that the umbral chromosphere oscillates with several co-spatial frequencies in the $5-8 \mathrm{mHz}$ band, but that the distribution of power varies from point to point within the umbra.

Another useful method to study non-stationary time series is a wavelet transform (e.g., Torrence \& Compo 1998) which calculates the power as a function of time and is able to localize different oscillation frequencies in a time interval in which they are present. An example of application of the wavelet transform to study chromospheric oscillations is given in Bocchialini \& Baudin (1995), and a more extended discussion of the wavelet transform in application to active region oscillations is given by Ireland et al. (1999).

We apply the wavelet analysis to the $\mathrm{O} \mathrm{V}$ time series from Fig. 3a, using a Morlet wavelet (for definition and details see, e.g., Torrence \& Compo 1998). Figure 5a shows the time series after subtracting the trend and the corresponding wavelet spectrum is shown in Fig. 5b. Only times greater than $50 \mathrm{~min}$ are displayed because the wavelet power was small for earlier times. The contour in Fig. 5b represents a $95 \%$ confidence level. The colour table is reversed and darker areas represent greater power. It can be seen that the period corresponding at any one time to the peak of the wavelet power varies with time. To demonstrate this better, the wavelet power has been normalised by dividing all powers at each time point by the value of the power peak at that time. Figure $5 \mathrm{c}$ shows how the period corresponding to the peak of the power spectrum varies with time. There is an obvious modulation of the period, ranging between 130 and $200 \mathrm{~s}$. For example, in the time interval $(75,87) \mathrm{min}$, where the power is strongest, the period increases from 160 to $200 \mathrm{~s}$. This period modulation is due either to temporal variability of the period within the $4^{\prime \prime}$ field of view of the instrument's slit or to spatial variability as the sunspot rotates by $2^{\prime \prime}$ during the $12 \mathrm{~min}$. 

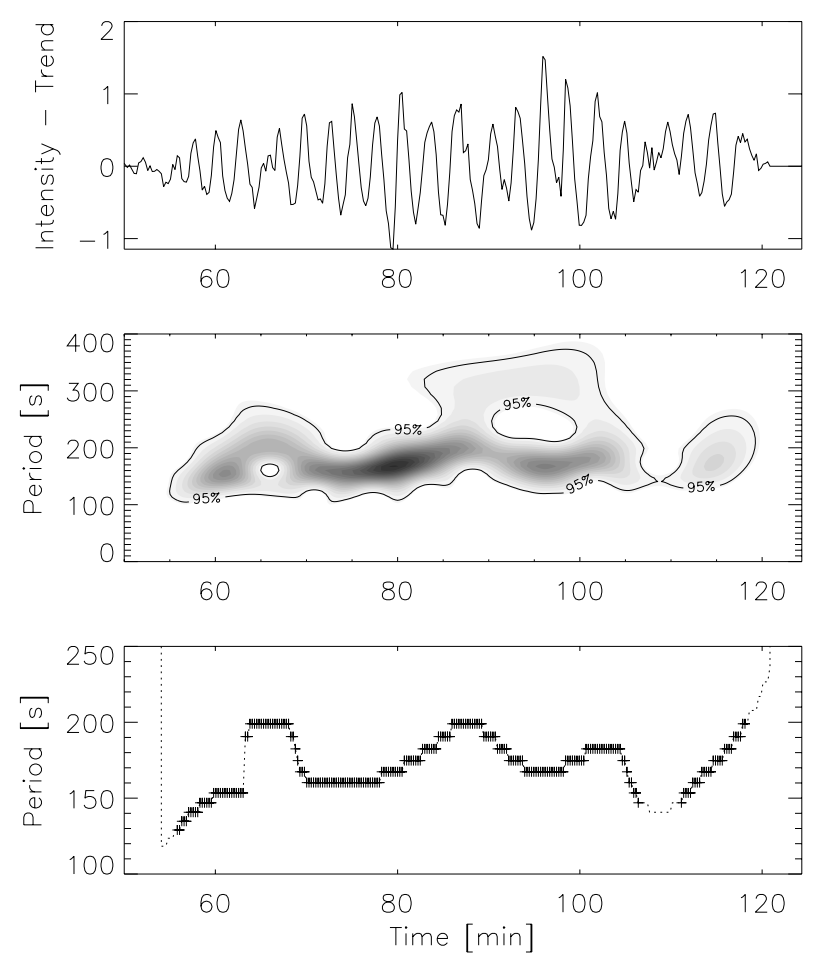

Fig. 5. Wavelet analysis of the sunspot oscillations in the O V $629.7 \AA$ line in NOAA active region 8369 on 30 October 1998. Line intensity has been summed over six brightest pixels $\left(10^{\prime \prime}\right)$ along the slit. a) Top panel: time history of the $\mathrm{O} \mathrm{V}$ intensity after subtracting the trend. b) Middle panel: wavelet power as a function of time and oscillation period. Darker areas denote greater power. c) Bottom panel: modulation of the oscillation period with time

The two peaks in the Fourier power spectrum in Fig. 4 have therefore been produced by the varying frequency of oscillation. The Fourier spectrum also shows a small peak near $3.3 \mathrm{mHz}$ corresponding to five minute oscillations. These oscillations can be identified in the wavelet power spectrum in the time interval $85-100 \mathrm{~min}$ in Fig. 5b.

\subsection{NOAA 8504, 3 April 1999}

Observations of the sunspot in NOAA active region 8504 on 3 April 1999 were made using solar tracking. The CDS slit was pointed slightly to the left of the brightest area in the sunspot plume and advanced by a $2^{\prime \prime}$ step every $12 \mathrm{~min}$. The pointing was kept fixed during each $12 \mathrm{~min}$ interval. The tracking rate $\left(10^{\prime \prime} /\right.$ hour $)$ was a little higher than the solar rotation rate of $7.5^{\prime \prime} /$ hour at the location of the sunspot $\left(-280^{\prime \prime},-370^{\prime \prime}\right)$. Because of that, the CDS slit moved in the same direction as the solar rotation but a little faster than the Sun, gradually overtaking the sunspot plume. The total distance covered by the slit in the E-W direction during 2.6 hours of observations is only 10.5 arcsec (including the $4^{\prime \prime}$ slit width) and spans the peak of the sunspot plume intensity.

Figure 6 shows the location of the sunspot plume relative to the sunspot. The contours of the umbra and penumbra have been derived from the SOHO/MDI white

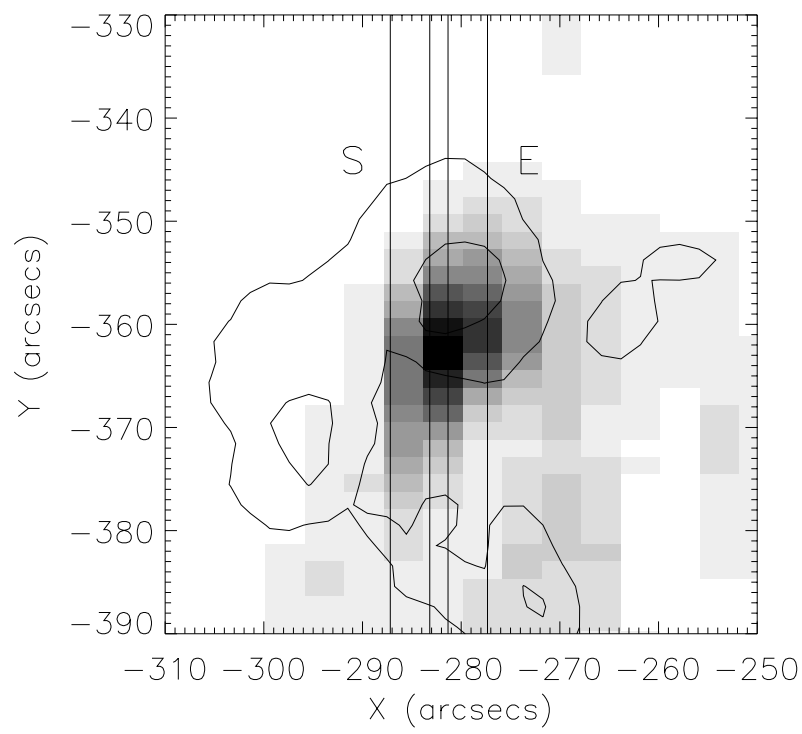

Fig. 6. A part of the CDS raster image of NOAA active region 8504 in the O V $629.7 \AA$ line on 3 April 1999. The colour table is reversed. The dark feature is a sunspot plume. The vertical lines show the CDS slit locations at the start ("S") and at the end ("E") of observation which was done using solar tracking for 2.6 hours. The contours of the sunspot's umbra and penumbra are indicated

light image taken at 04:47:33 UT, rotated to the time of the CDS observation of the sunspot plume at 07:13:32 UT. The pointing of the CDS raster has been adjusted by correlating the He I $584 \AA$ raster with the SOHO/EIT He 304 image taken at 07:25:53 UT.

This observation used a different selection of lines: He I 584.3 A, O IV 554.5, O V 629.7, Ne VI 562.9, and $\mathrm{Mg}$ IX $368.06 \AA$. The sunspot plume was clearly seen in the O IV, O V and Ne VI lines $\left(1.7-410^{5} \mathrm{~K}\right)$. It was much less intense in the chromospheric He I line, and not seen in the coronal Mg IX line at all. Strong oscillations were seen only in the O IV and $\mathrm{O}$ V lines while the oscillation amplitude was much lower in the Ne VI line.

The time history of the intensity in the O V $629.7 \AA$ line, summed over 17 arcsec along the slit, is shown in Fig. 7a. A linear trend was fitted separately to each $12 \mathrm{~min}$ interval and subtracted from the intensity curve. The resulting curve is shown in Fig. 7b. The Fourier power spectrum of the time series is shown in Fig. 8. In contrast to Fig. 4, this power spectrum has only one dominant peak with the frequency of $5.9 \mathrm{mHz}(170 \mathrm{~s}$ period), although other frequencies are also present in the range $5-8 \mathrm{mHz}$ with a statistically significant power. The wavelet power of the $\mathrm{O} \mathrm{V}$ time series in the time interval $(17,133) \mathrm{min}$, where clear oscillations are seen, has a rather constant period, consistent with the dominance of one frequency in the Fourier power spectrum.

The time series taken in the O IV $554.5 \AA$ line, formed around $1.710^{5} \mathrm{~K}$, has a similar power spectrum. The maximum amplitude of the O IV oscillations is 10 percent, a little greater than that of the $\mathrm{O} \mathrm{V}$ line (8 percent). 

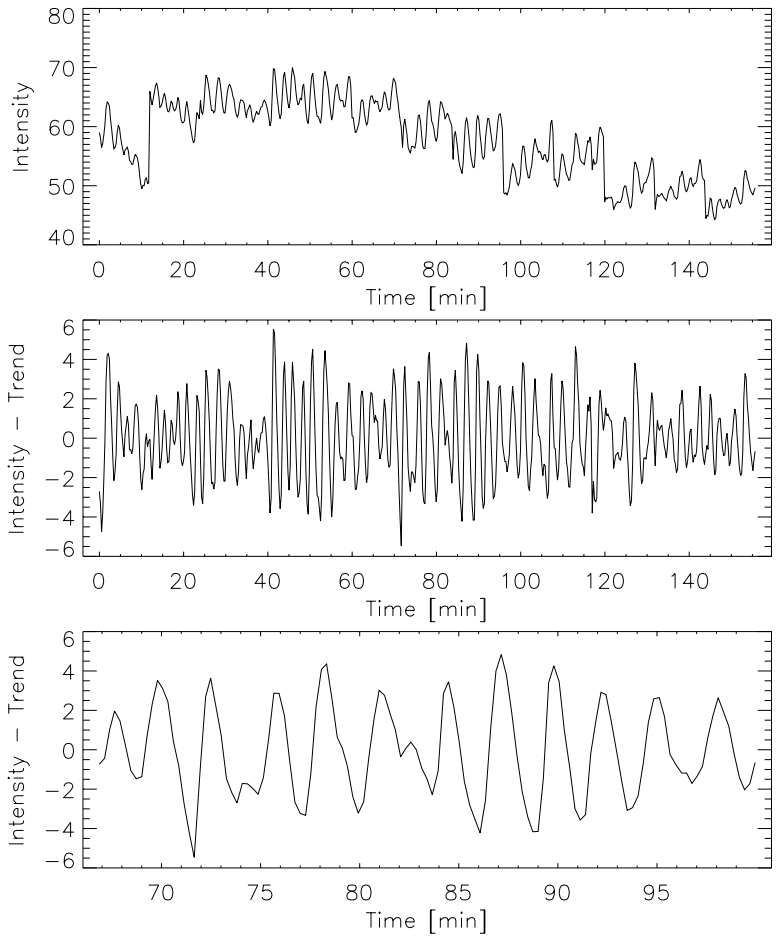

Fig. 7. Oscillations above a sunspot in NOAA active region 8504 on 3 April 1999. The pointing was advanced in the direction of solar rotation by a $2^{\prime \prime}$ step every $12 \mathrm{~min}$. a) Top panel: time history of the O V $629.7 \AA$ line intensity integrated over the sunspot plume area. b) Middle panel: the $\mathrm{O} V$ line intensity after subtracting the linear trend from each 12 min sequence. c) Bottom panel: expanded time interval $(66,100) \mathrm{min}$

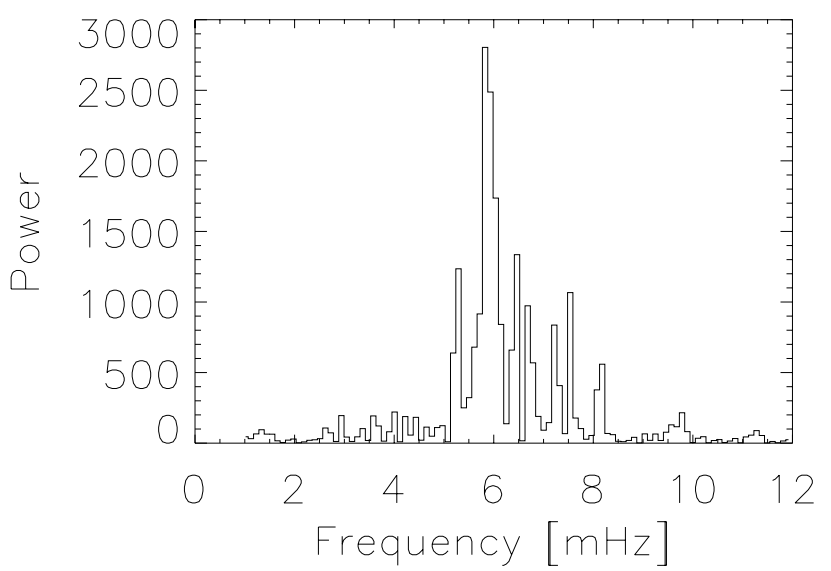

Fig. 8. Fourier power spectrum of the sunspot oscillations in the O V $629.7 \AA$ line intensity in NOAA active region 8504 on 3 April 1999

The oscillation amplitudes vary with time by about a factor of two.

The intensity of the Ne VI line $\left(410^{5} \mathrm{~K}\right)$ has a rather noisy time series with a very small amplitude of oscillations, but the power spectrum shows a noticeable bump between 4 and $7 \mathrm{mHz}$, with a peak around $6 \mathrm{mHz}$, and then another narrower bump with a peak around $8.3 \mathrm{mHz}$. This shows that the oscillations reach the $410^{5} \mathrm{~K}$ plasma.

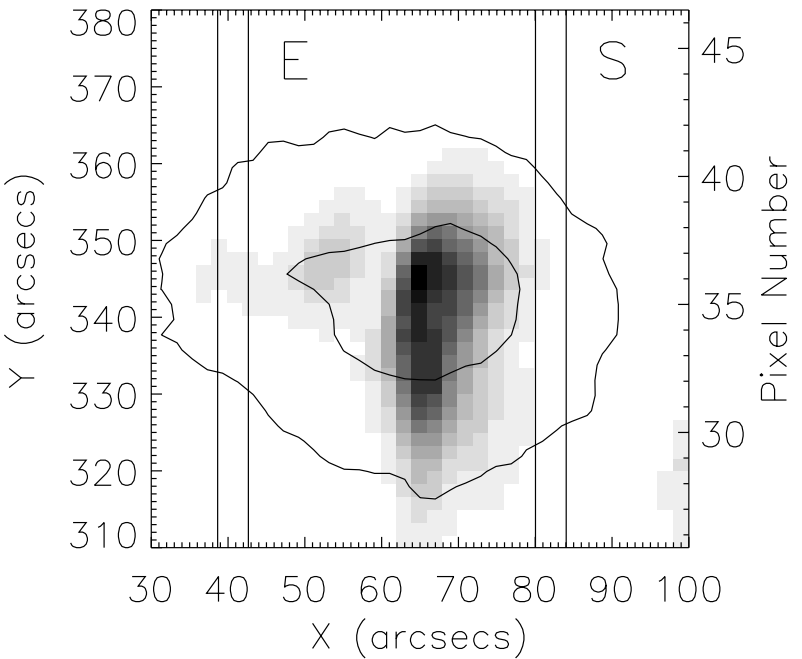

Fig. 9. A part of the CDS raster image of NOAA active region 8552 in the O V $629.7 \AA$ line on 30 May 1999. The colour table is reversed. The dark feature is a sunspot plume. The vertical lines show the CDS slit locations at the start ("S") and the end ("E") of the analysed time series. The contours of the sunspot's umbra and penumbra are indicated. Pixel numbers on the right-hand side correspond to double-binned pixels shown in Figs. 10 and 18

In the same ten pixels, the intensity of the chromospheric line of He I $584 \AA$ has very small oscillations, and an unusual power spectrum consisting of narrow peaks at 1.6, 4.6, 5.9, and $6.6 \mathrm{mHz}$.

\subsection{NOAA 8552, 30/31 May 1999}

A sunspot in NOAA active region 8552 was observed on two days, 30 and 31 May 1999. The first observation on 30 May had fixed pointing, while the second observation, on 31 May, used tracking.

\subsubsection{May 1999, fixed pointing}

The observing sequence consisted of a $2^{\prime} \times 4^{\prime}$ raster in several transition region lines taken between 11:10:43 UT and 11:37:04 UT, followed by a 6.5 -hour observation using a $4^{\prime \prime} \times 240^{\prime \prime}$ slit which was pointed to the right of the sunspot's penumbra and kept at that position while the sunspot moved in front of the slit with solar rotation. The following spectral lines were used: He I $584 \AA$, O III 599, O IV 554, O V 629.7, and Ne VI $562 \AA$. Pixels along the slit were double-binned, giving the spatial resolution of $4^{\prime \prime}$ in the horizontal direction and $3.4^{\prime \prime}$ in the vertical direction. A total of 1500 exposures were made, out of which 1000 exposures, starting at 13:47:46 UT, show the presence of oscillations and have been selected for analysis.

The emission in the transition region line of O V $629.7 \AA$ shows a bright area which is either one sunspot plume with a complex geometry or two sunspot plumes close together. Figure 9 shows the location of the sunspot plume with respect to the sunspot's umbra and 


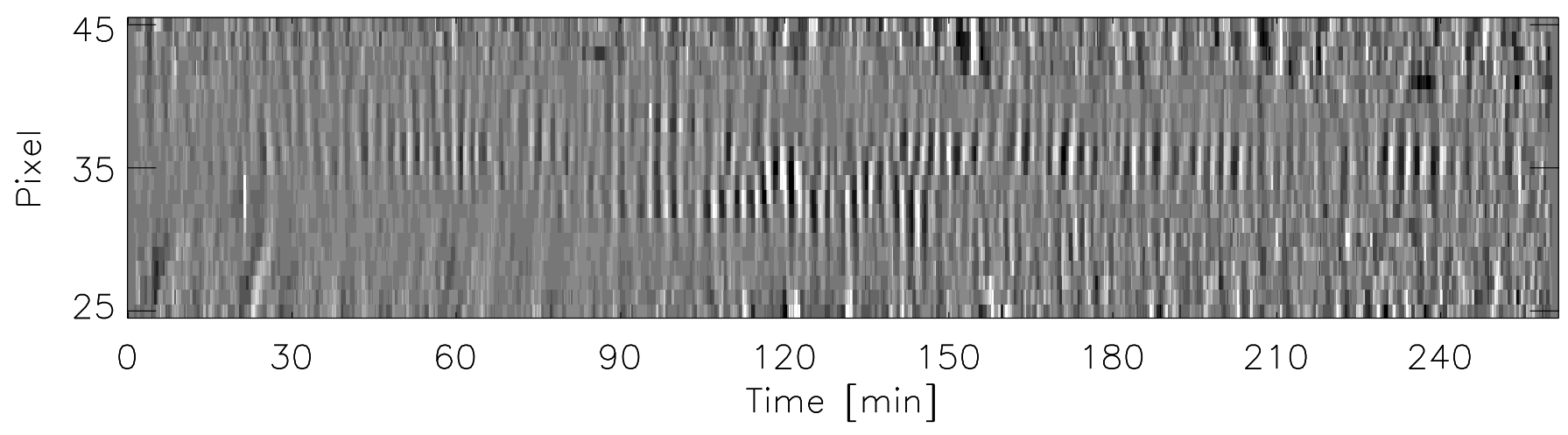

Fig. 10. Time variability of the intensity of the O V $629.7 \AA$ line along the CDS slit, during the observation of NOAA 8552 on 30 May 1999. The scale along the $Y$ axis is pixel number. Pixel size is $3.36^{\prime \prime}$. The trend has been divided into each pixel's time history

penumbra. The sunspot's contours have been drawn from the SOHO/MDI white light image taken at 11:11:33 UT, rotated to the time of observation of the sunspot plume in the CDS raster (11:31:08 UT). The CDS pointing has been adjusted by correlating the He I $584 \AA$ raster at 11:10:43 UT UT with the EIT He $304 \AA$ image taken at 13:18 UT (the solar rotation between the two times has been taken into account).

The time series for this sunspot differs in one important way from the previously described sunspots. Here, the oscillations are seen both in the area of high intensity in the large sunspot plume and in another area separate from the plume where the $\mathrm{O} \mathrm{V}$ line intensities are at least four times lower.

Figure 10 shows the oscillations along a part of the CDS slit, in the same $70^{\prime \prime}$-long area between pixels 25 to 45 as shown in Fig. 9. The signal has been divided by the trend, to show better the oscillations in the weak areas outside the sunspot plume. Below we analyse data for two selected pixels. Pixel number 36 cuts through a large sunspot plume in the time interval 30-130 min and through an oscillating area of lower intensities to the left of the sunspot plume in the time interval 140-250 min (located between $X=40^{\prime \prime}-55^{\prime \prime}$ and $Y=342^{\prime \prime}-346^{\prime \prime}$ in Fig. 9). Pixel number 32 (between $Y=330^{\prime \prime}$ and $Y=334^{\prime \prime}$ in Fig. 9) shows significant oscillations only when it crosses the lower end of the large plume.

Figure 11a shows the O V time series from pixel number 36 and Fig. 11b shows this series after subtracting the trend. Prominent oscillations are seen across the sunspot plume. When the signal from Fig. 11b is divided by the trend, it is clearly seen in Fig. 12a that the relative oscillation amplitudes in the low-intensity area outside the sunspot plume are even greater than in the sunspot plume, reaching up to $14 \%$.

Figure $12 \mathrm{~b}$ shows the wavelet power of the time series from Fig. 12a. Figure 12c shows the time variability of the period corresponding to the maximum wavelet power at each time point. The crosses show time intervals where the power is greater than 10 percent of the global maximum of the wavelet power, and the dotted line shows where the
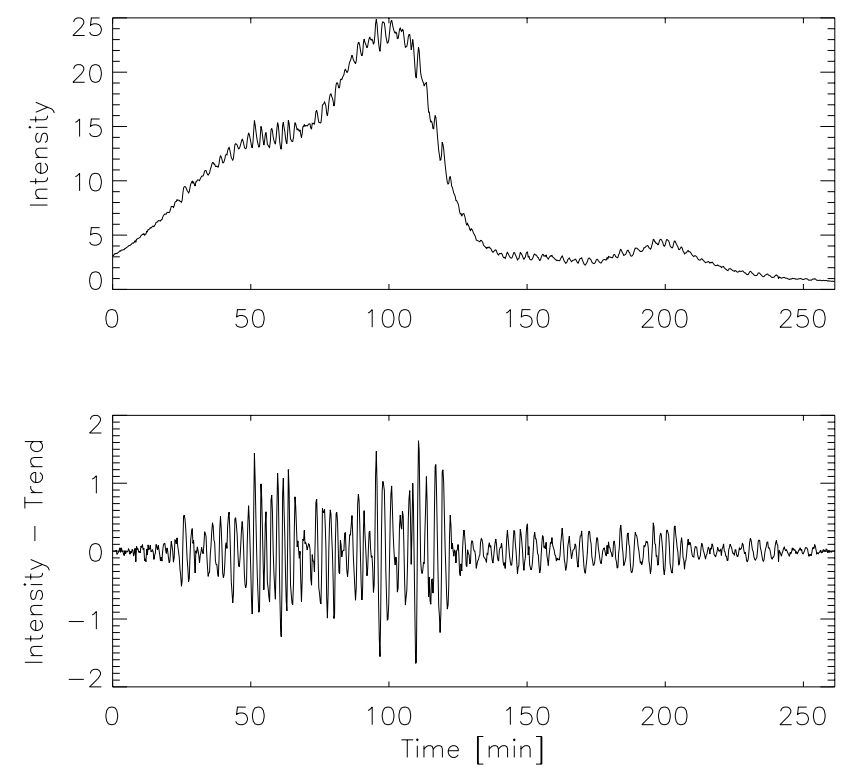

Fig. 11. Sunspot plume observations in NOAA active region 8552 on 30 May 1999. The pointing was kept fixed during the observation. a) Top panel: time history of the O V $629.7 \AA$ line intensity in pixel number 36 . b) Middle panel: the $\mathrm{O} V$ line intensity after subtracting the trend

power is less than the 10 percent threshold. It can be seen that the period fluctuates in the range 120-210 s.

The Fourier power spectrum for pixel 36 was calculated separately for the sunspot plume (Fig. 13) and the fainter area (Fig. 14). Both power spectra show more frequencies than those for the previous sunspots. The main difference between the sunspot plume and the weaker-intensity area is an additional component at $8 \mathrm{mHz}$ seen in the sunspot plume. This agrees with the wavelet power spectrum which shows periods around $120 \mathrm{~s}$ in the sunspot plume in the time interval 53-65 $\mathrm{min}$.

A time series for pixel 32 around the sunspot plume is shown in Fig. 15a after subtracting the trend. Figure 15b shows the same time series divided by the trend. The Fourier power spectrum of the entire time series in pixel 32 , for the entire $260 \mathrm{~min}$ of observation, is shown in Fig. 16. This power spectrum differs from the power 

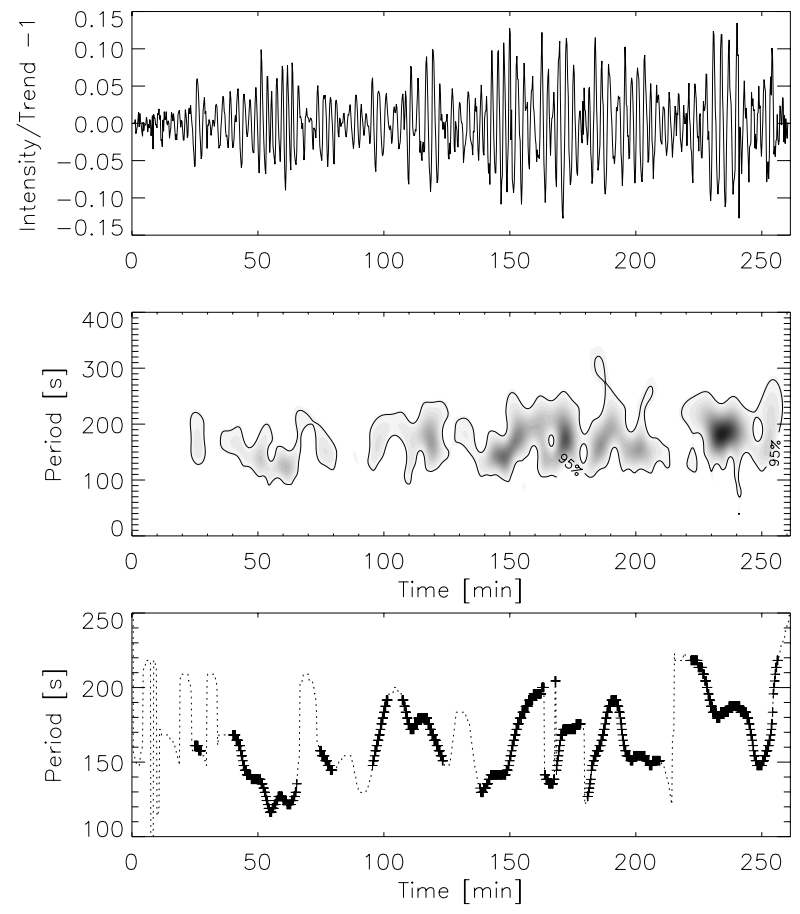

Fig. 12. Wavelet analysis of the sunspot oscillations in the O V $629.7 \AA$ line in NOAA active region 8552 on 30 May 1999. a) Top panel: time history of the O V $629.7 \AA$ line intensity in pixel number 36 divided by the trend, minus 1 . b) Middle panel: wavelet power for the time series from the top panel, as a function of time and the oscillation period. Darker areas denote greater power. c) Bottom panel: modulation of the oscillation period with time

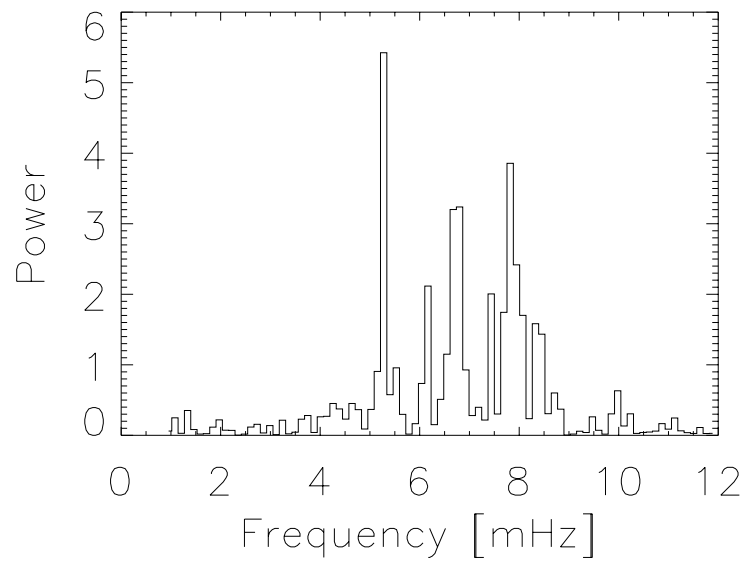

Fig. 13. Fourier power spectrum for pixel number 36 for the O V time series on 30 May 1999, in the high intensity area in the time interval $(0,130)$ minutes

spectrum in pixel 36 by concentration of most of the power between 5 and $6 \mathrm{mHz}$.

A summary of the oscillatory behaviour of the $\mathrm{O} \mathrm{V}$ intensity in each pixel along the slit is given in Fig. 17a, which shows the Fourier power spectrum as a function of frequency and pixel number.

The O IV line $\left(\begin{array}{llll}1.7 & 10^{5} \mathrm{~K}\end{array}\right)$ shows oscillations with a slightly larger relative amplitude than the $\mathrm{O} \mathrm{V}$ line. The

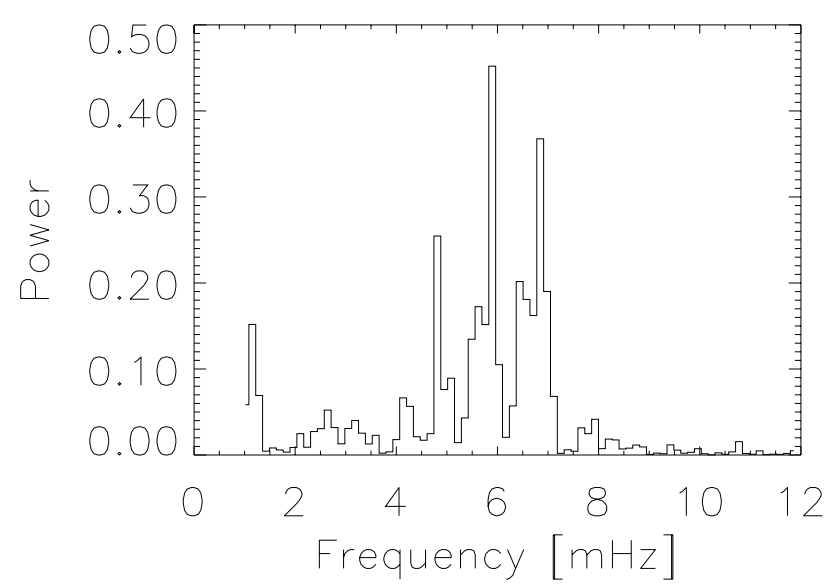

Fig. 14. Fourier power spectrum for pixel number 36 for the O V time series on 30 May 1999, in the low intensity area in the time interval $(138,260)$ minutes
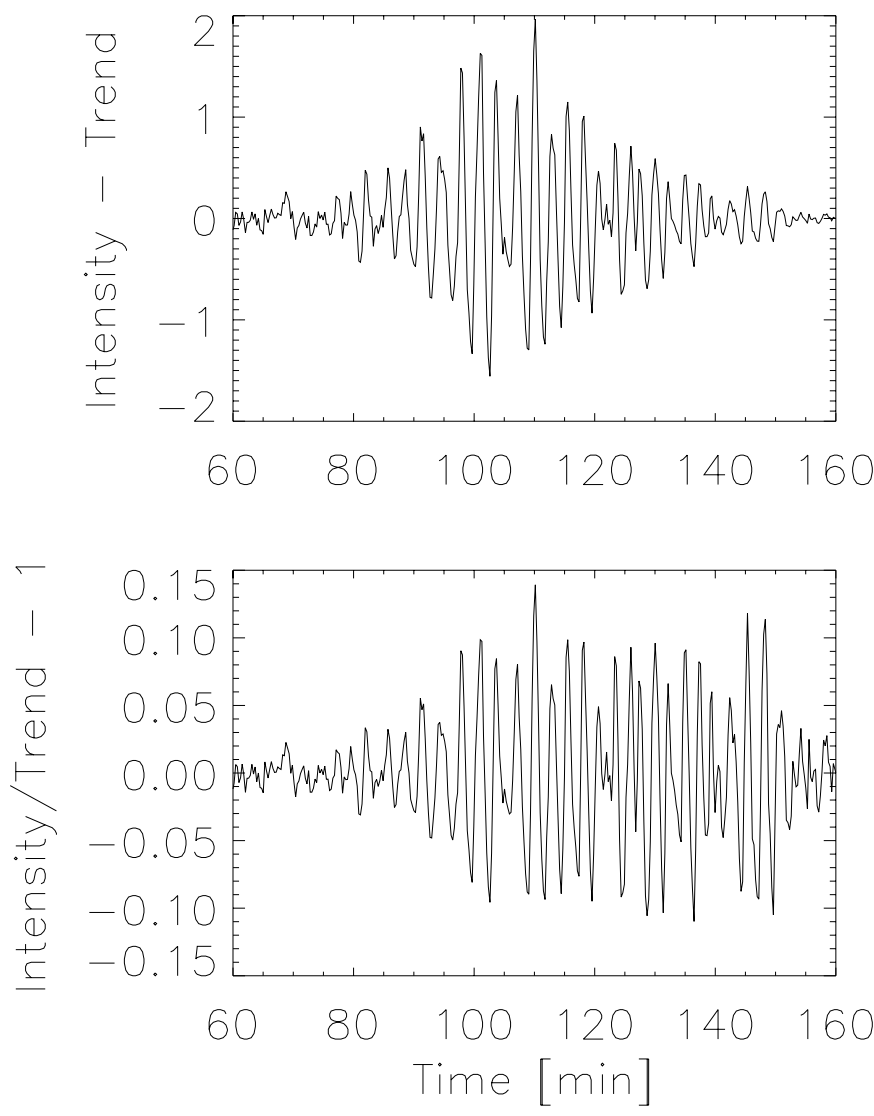

Fig. 15. Time series of the O V line intensity in pixel 32 near the sunspot plume in NOAA active region 8552 observed on 30 May 1999. a) Top panel: the trend has been subtracted from the intensity, b) Bottom panel: the series from the top panel has been divided by the trend

O IV power spectrum is very similar to the $\mathrm{O} V$ power spectrum in pixel number 36 .

The O III (9 $\left.10^{4} \mathrm{~K}\right)$ and Ne VI $\left(\begin{array}{lll}4 & 10^{5} \mathrm{~K}\end{array}\right)$ lines are weaker and the emission in these lines is seen well only near the peak of the sunspot plume. Oscillations are detectable in both of these lines, but the oscillation amplitudes are lower and the time series are noisier. The Ne VI 


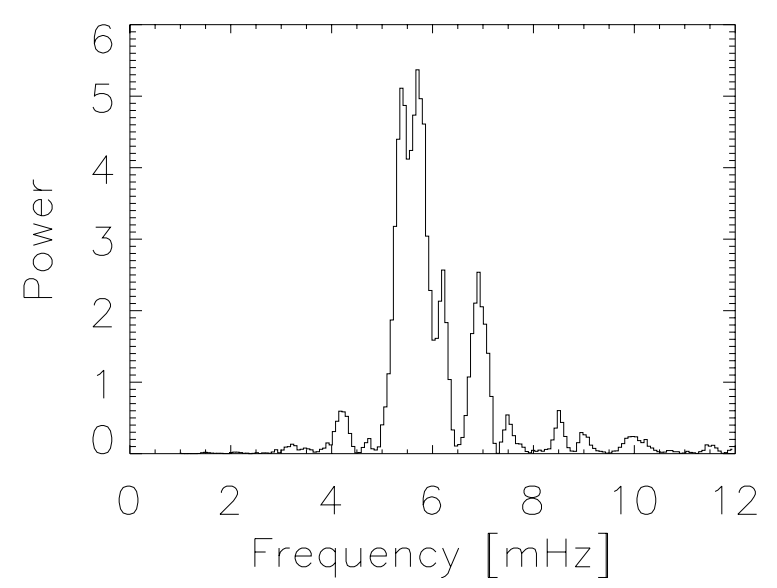

Fig. 16. Fourier power spectrum for pixel number 32 for the O V time series on 30 May 1999, in the time interval $(0,261)$ minutes
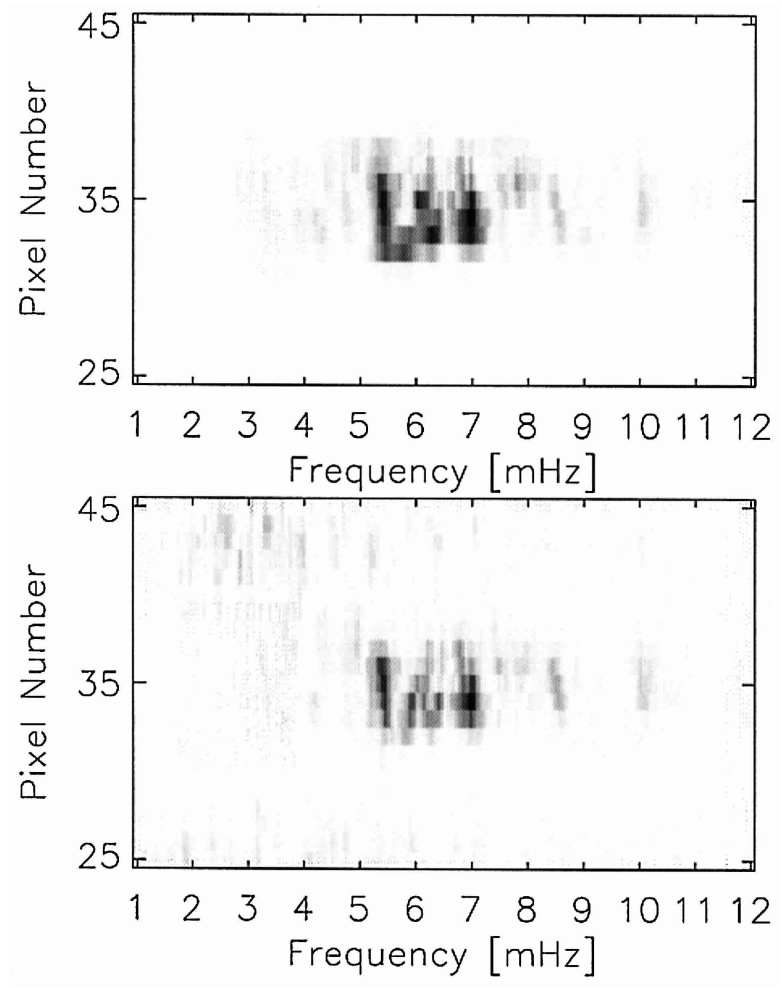

Fig. 17. A map of the Fourier power spectrum for 20 doublebinned pixels along the slit on 30 May 1999: a) derived from the O V $629.7 \AA$ intensity series (top panel), b) derived from the He I $584.3 \AA$ intensity series (bottom panel). The Fourier spectrum was taken for each pixel separately, from the entire $260 \mathrm{~min}$ time series. The spatial pixel size is $3.36^{\prime \prime}$

line Fourier power spectrum for pixel number 36 has a dominant peak in the range $5.0-5.5 \mathrm{mHz}$, with a maximum around $5.2 \mathrm{mHz}$. Higher frequencies have very low power, and the power spectrum is noisier.

As noted by Fludra et al. (1997), in contrast to the transition region lines, the chromospheric He I $584.3 \AA$ line does not show intensity enhancements in sunspot plumes. The He I intensity above the umbra is comparable to, and often less bright than other chromospheric features

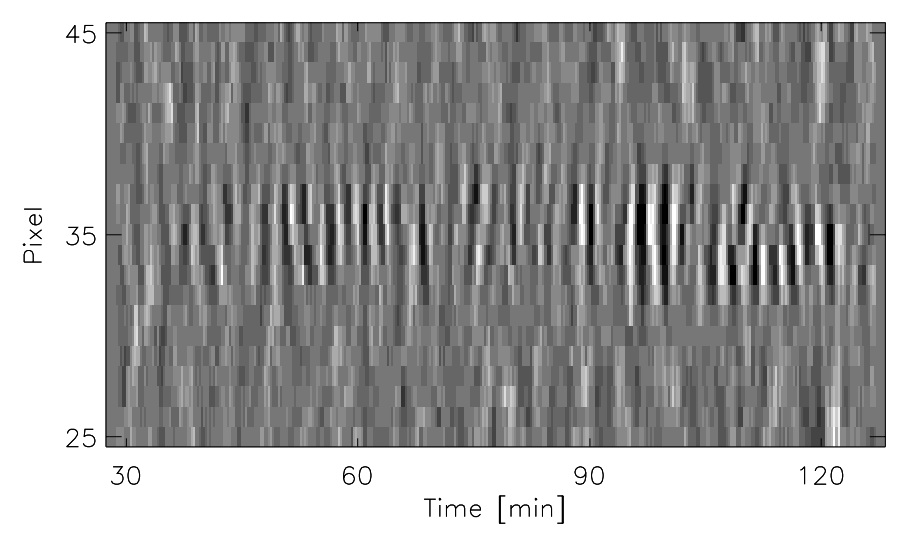

Fig. 18. Time variability of the intensity of the He I $584 \AA$ line along the CDS slit, during the observation of NOAA 8552 on 30 May 1999. The scale along the $Y$ axis are pixel numbers, as in Fig. 9. A trend has been divided into each pixel's time history. Pixel size is $3.36^{\prime \prime}$

outside the sunspot. However, we find that the intensity of the He I $584.3 \AA$ line oscillates in the umbra below the sunspot plume in NOAA active region 8552, as shown in Fig. 18. The He I intensity in pixel 36 has the same frequency components in the power spectrum as the $\mathrm{O} V$ series. The He I line also oscillates in the faint area outside the plume but with a lower relative amplitude (whereas the O V $629.7 \AA$ line at the same location outside the plume oscillates with a higher relative amplitude than in the plume). The map of the Fourier power spectrum for the He I $584.3 \AA$ line, shown in Fig. $17 \mathrm{~b}$, is very similar to the $\mathrm{O} \mathrm{V}$ power spectrum map in Fig. 17a.

The peak of this sunspot plume's intensity oscillates most regularly. It must be stressed, however, that not all locations within the sunspot plume oscillate. There may be areas of both high and low intensity which are part of the sunspot plume and which do not show oscillations.

This sunspot presents a more complex power spectrum, with a larger number of frequencies than in the spectra of two sunspots described in the previous sections and one sunspot described by Fludra (1999a). While this is more difficult to interpret, the advantage of this observation is that it shows oscillations at two locations, in a bright sunspot plume above the umbra and in a separate area above an umbral extension, much weaker than the plume but still enhanced over the rest of the surrounding transition region. We also point out the indication of intermittent wave activity outside the sunspot area which can be partially seen in Figs. 10 and 18, with frequencies in the range $1.6-4.5 \mathrm{mHz}$.

\subsubsection{May 1999, solar tracking}

The same sunspot in NOAA 8552 was observed again on 31 May 1999, this time using solar tracking. The slit was placed across the sunspot plume (see Fig. 19) and then advanced by $4^{\prime \prime}$ every $27 \mathrm{~min} 40 \mathrm{~s}$. Thus, the tracking rate was $8.7^{\prime \prime}$ / hour, slightly higher than the solar rotation rate of $8.0^{\prime \prime} /$ hour at the sunspot's location $\left(320^{\prime \prime}, 330^{\prime \prime}\right)$. During 


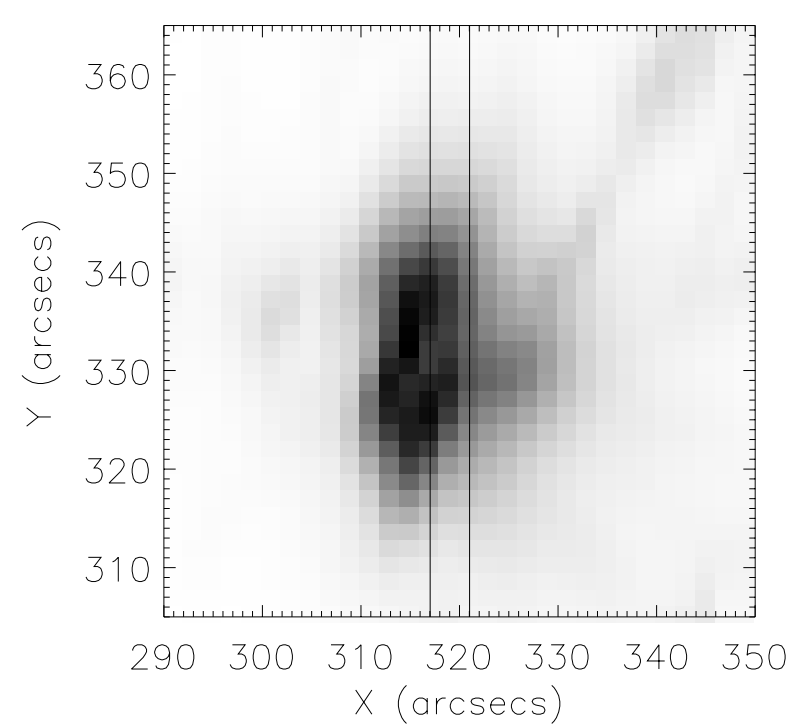

Fig. 19. A part of the CDS raster image of NOAA active region 8552 in the O V $629.7 \AA$ line on 31 May 1999. The colour table is reversed. The dark feature is a sunspot plume. The vertical lines show the CDS slit location at the beginning of observation which was done using solar tracking for 3.4 hour. The sunspot contours are not shown because EIT images were not available on 31 May 1999 for the CDS pointing adjustment

3 hours and 25 min of observation, the slit overtook the sunspot by only $2.4^{\prime \prime}$. During each $27.6 \mathrm{~min}$ interval the slit was kept at a fixed position and the total extent covered by the slit in the E-W direction was approximately $8^{\prime \prime}$ ( $4^{\prime \prime}$ slit width plus $4^{\prime \prime}$ of solar rotation).

We have derived a time series by summing five doublebinned pixels covering $17^{\prime \prime}$ along the slit. The time series in the $\mathrm{O} \mathrm{V}$ line is shown in Fig. 20a after subtracting a linear background from each $27.6 \mathrm{~min}$ segment. The wavelet power and the peak of the power spectrum in the time-period coordinates are shown in Figs. 20b and c, respectively. The variability of the period is smaller than in Fig. 12. Most of the time the period is in the range 150-190 s.

he Fourier power spectrum in Fig. 21 was calculated for the first 105 min (420 exposures) of the $\mathrm{O} \mathrm{V}$ time series and it shows a dominant peak at $5.6 \mathrm{mHz}$. Clearly, the solar tracking reduced the spread of frequencies compared to the observation on 30 May.

Intensities of all three lines, He I, O IV, and O V, show nearly identical oscillations: the O IV line shows strong oscillations with the same power spectrum as the $\mathrm{O} \mathrm{V}$ line, and amplitudes up to 12 percent. The He I line has equally strong oscillations with power concentrated between 5 and $7 \mathrm{mHz}$, peaking at $5.7 \mathrm{mHz}$, and amplitudes of 12 percent. Only the Ne VI line has a very low amplitude and a noisy time series, but the power spectrum still shows a narrow peak at $5.7 \mathrm{mHz}$.
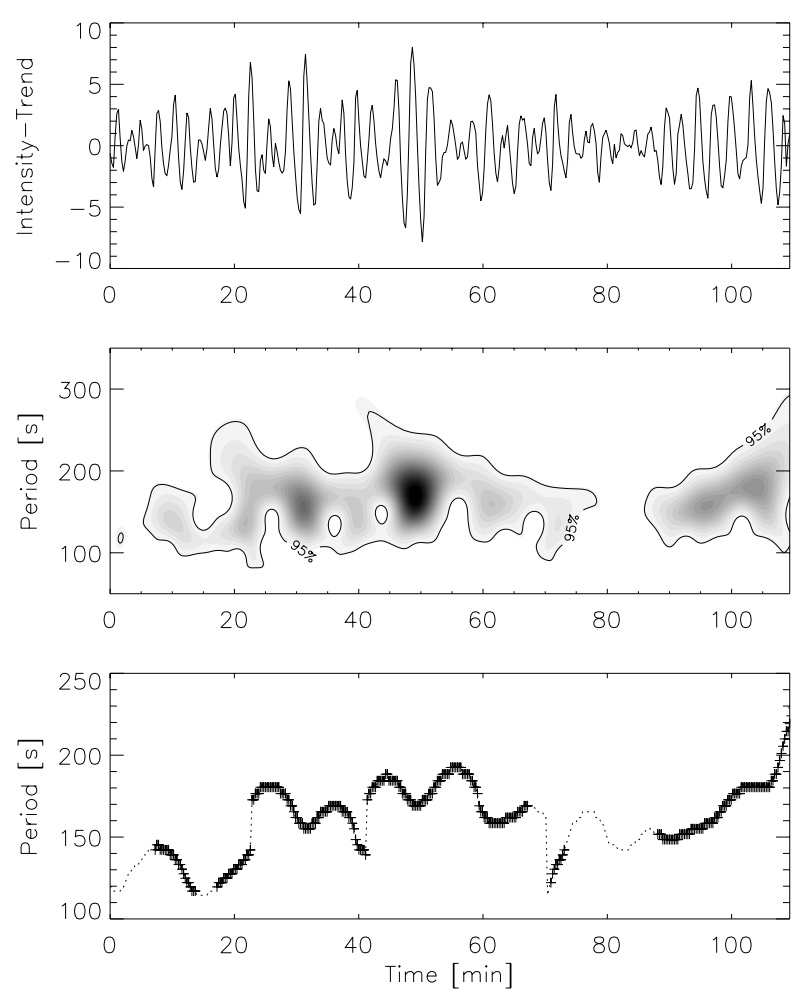

Fig. 20. Wavelet analysis of the sunspot oscillations in the O V $629.7 \AA$ line for five summed pixels $\left(17^{\prime \prime}\right)$ in NOAA active region 8552 on 31 May 1999. a) Top panel: time history of the $\mathrm{O} \mathrm{V}$ line intensity with the trend subtracted. b) Middle panel: wavelet power for the time series from the top panel, as a function of time and the oscillation period. Darker areas denote greater power. c) Period corresponding to a maximum power at each time point (bottom panel)

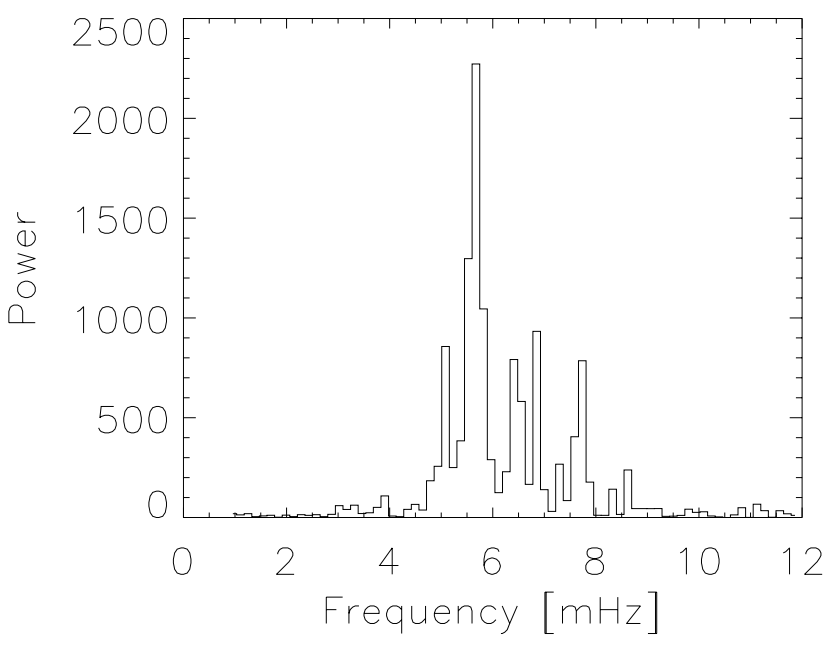

Fig. 21. Fourier power spectrum for the first 420 exposures (109 min) of the time series of O V line intensities on 31 May 1999 (obtained with solar tracking). The trend was subtracted from the series before calculating the power spectrum

\section{Discussion}

The nature of the transition region oscillations has been discussed by Brynildsen et al. (1999a) who measured sunspot transition region oscillations both in velocity and 
intensity using SUMER data, and concluded that their measurements were consistent with upward propagating acoustic waves. We assume that the oscillations in intensity we study are caused by the same type of waves. In this paper, we take advantage of the unique features of our observations - long time series and the use of the solar tracking - to address further questions: resolving different components of the power spectrum, spatial and temporal variability of the oscillation period, and the consequences of the apparent localisation of the oscillations near the regions of increased intensity in sunspot plumes.

\subsection{Variability of the period}

Because of the long time series obtained, particularly those that use solar tracking, we have achieved a very good frequency resolution in the Fourier power spectrum. The power spectra in different sunspots show a variety of cases. For time series obtained with fixed pointing, we have found sunspots that have two peaks in the power spectrum, with close periods between 5.5 and $6.3 \mathrm{mHz}$ (see Fig. 4, and also Fig. 2 in Fludra 1999a). Other sunspots can have more complex power spectra with several peaks spread over a wider frequency range, between 4 and $8 \mathrm{mHz}$ (see Figs. 13 and 14). Larger periods, 200-250 s, tend to be associated with lower oscillation amplitude, when the oscillations temporarily die down. Only in one case so far have we found a transient presence of five minute oscillations.

The Fourier power spectrum tells us whether a particular frequency component exists in the time series or not, but when applied to the whole series it cannot tell where in time this component exists or what its duration is. The Fourier transform can still be used in nonstationary time series, i.e., time series with frequency components varying in time, if we only want to know what frequency components exist. However, if we would also like to know precisely when the different frequency components are present, another method is needed.

We have applied the wavelet analysis to locate these frequency components in time. From the wavelet power spectra in Figs. 5, 12, and 20 it can be seen that the period corresponding to the peak of the power spectrum fluctuates during the observation. At certain times there is an abrupt increase of the period, taking place over the duration of one exposure $(15 \mathrm{~s})$. Those cases can be attributed to a temporal change in the oscillation period. At other times the period changes gradually on timescales of up to $15 \mathrm{~min}$. For example, the period increases from $140 \mathrm{~s}$ to $190 \mathrm{~s}$ during approximately $10 \mathrm{~min}$ of observation between $t=144 \mathrm{~min}$ and $t=154 \mathrm{~min}$ in Fig. 12. In another instance, the period decreases from $160 \mathrm{~s}$ to $130 \mathrm{~s}$ during $15 \mathrm{~min}$, between $t=39 \mathrm{~min}$ and $t=55 \mathrm{~min}$. In 10-15 min, the sunspot has rotated by $1.5-2$ arcsec, which is up to 50 percent of the slit width. This period variability could be due to a spatial change, i.e., different frequencies present at different spatial locations, or it could represent a slow temporal change.

When the same sunspot in NOAA 8552 is observed with tracking on 31 May 1999, the Fourier spectrum also shows several frequencies but the spread of frequencies has been reduced and the frequency around $5.7 \mathrm{mHz}$ has become dominant. The wavelet transform also shows that the fluctuations of the oscillation period are smaller in this case. Therefore, the multitude of peaks in the power spectrum and the slow variability of the period seen in the 30 May 1999 dataset without solar tracking (see Sect. 3.3.1) is likely caused by the spatial change of the oscillation period. Another observation made with solar tracking, of NOAA 8503 on 3 April 1999, also shows one dominant peak at $5.8 \mathrm{mHz}$.

From these two examples made with solar tracking it appears that following one location in the sunspot tends to bring out one dominant frequency near $5.8 \mathrm{mHz}$ and reduce the power in higher frequencies. The fact that other frequencies are not eliminated completely could be explained by the fact that even with tracking the covered area is at least $6^{\prime \prime}$ (the $4^{\prime \prime}$ slit width plus $2^{\prime \prime}$ solar motion in each 12 min period between the slit advancement). In addition, there is a contribution to the line intensity from a somewhat larger area since the instrument spatial response function has a few arcsecond width (we discuss this in Sect. 4.2).

From the comparison of the Fourier and wavelet power spectra we conclude that most of the different peaks seen in the Fourier power spectra do not represent frequencies that are present simultaneously at one spatial location, but rather that these frequencies appear in different parts of the time series. This finding may have important consequences for the interpretation of all past data sets measuring sunspot oscillations and analysed before the recent advent of the wavelet analysis.

One comment about the wavelet transform is that it has a local nature in the time domain and the calculation of the wavelet power in a specific time interval does not take into account the whole history of the time series before and after this interval. Therefore, for example, if the envelope of the amplitude of the time series reaches zero due to interference of two periodic signals with close oscillation periods, the wavelet power may also be zero near this point. Low values of the wavelet power only reflect the local low amplitudes of the time series but do not necessarily indicate the absence of underlying components that add up to create the time series.

\subsection{Spatial localisation of the oscillations}

In all three sunspots examined in this paper and in the sunspot examined by Fludra (1999a), the oscillations seen in the CDS data appear to coincide spatially, to within the spatial resolution of the instrument, with the increase of intensity in a sunspot plume. Below we consider consequences and interpretations of this observation. 
(1) The first obvious possibility to consider is that the plasma in the sunspot plume is primarily responsible for the oscillations of the high-intensity areas seen by CDS. In this case, the relative amplitude of the intensity oscillations in the sunspot plumes would be 5-10 percent, with occasional peaks up to 15 percent, as observed in Figs. 3, 7, 12a, and 15b. However, an independent measurement of the Fourier power spectrum made in other sunspots by the SUMER instrument (Brynildsen et al. 2000) with a better spatial resolution of $1^{\prime \prime}$, suggests that the oscillation power is concentrated in lower intensity areas in the umbra, adjacent to the sunspot plumes. While these two different results were obtained for different sunspots, CDS tends to see the oscillations associated with nearly all sunspot plumes and the difference between CDS and SUMER results most likely stems not from the sunspots themselves but from the interpretation of data from one of the instruments, i.e., either the CDS observation or the SUMER observation needs to be explained differently. One possible instrumental effect that might cause SUMER to record diminished oscillations in sunspot plumes would be a saturation of the detector. If due to the high intensities in bright sunspot plumes SUMER detectors operated on a very non-linear part of their intensity response, the relative oscillation amplitude would be suppressed in sunspot plumes. Figure 3 in Wilhelm et al. (1997) shows that the countrates seen in sunspot plumes can cause some detector gain depression, however, the effect does not seem severe. We will therefore consider the other possibility, i.e., a different interpretation of the CDS observation.

(2) Comparison of the area of the sunspot plume seen in the CDS and SUMER instruments, shown for three sunspots by Brynildsen et al. (2000), shows that plumes appear more compact in SUMER data than in CDS data. This is consistent with the measurement of the spatial response of the CDS normal incidence spectrometer made by Pauluhn et al. (1999) which gives a FWHM of $6^{\prime \prime}$ and $8^{\prime \prime}$ for the $X$ and $Y$ directions, respectively. It is therefore expected that at any given location along the CDS slit there is a contribution to the line intensity coming not only from inside the area viewed by each raster element (pixel) but also from the areas adjacent to the pixel, within a few arcseconds around it. In that case, if the source of the oscillations were close to the sunspot plume but separate from it, the CDS would register a combined intensity from the oscillation source and the sunspot plume.

Let us assume that this is the case and examine the consequences. We will tentatively suppose that the plasma at transition region temperatures in sunspot plumes does not oscillate, and only the low-intensity plasma in the area adjacent to the plume oscillates. The CDS instrument would then see a superposition of the two intensities - a high intensity $I_{\mathrm{p}}$ in the non-oscillating plume and a low background intensity $I_{\mathrm{b}}$ of the oscillating plasma above the umbra. At the peak of the recorded intensity curve, the total intensity $I_{\mathrm{p}}+I_{\mathrm{b}}$ is typically $5-10$ times greater than the background non-plume intensity $I_{\mathrm{b}}$, and the maximum relative observed amplitude $\Delta I /\left(I_{\mathrm{p}}+I_{\mathrm{b}}\right)$ reaches $10-15$ percent. Taking $\Delta I_{\mathrm{b}}=\Delta I$ and conservative values of $\left(I_{\mathrm{p}}+I_{\mathrm{b}}\right) / I_{\mathrm{b}}=5$ and $\Delta I /\left(I_{\mathrm{p}}+I_{\mathrm{b}}\right)=0.1$ we obtain $\Delta I_{\mathrm{b}} / I_{\mathrm{b}}=0.5$. Therefore, the low intensity plasma would have to oscillate with a very high relative amplitude $\Delta I_{\mathrm{b}} / I_{\mathrm{b}}$ greater than 50 percent.

To recapitulate the two possibilities of interpreting the oscillations of the high-intensity areas seen in the CDS data: either (1) the high-intensity plasma in sunspot plumes oscillates with relative amplitudes $5-10$ percent, or (2) if the sunspot plumes do not oscillate at all, then the low-intensity plasma must oscillate with relative amplitudes greater than 50 percent, and the source of these oscillation is always located within a few arcseconds from the bright parts of sunspot plumes.

To see if the second interpretation agrees with the SUMER data, we turn again to the SUMER observations reported by Brynildsen et al. (2000) to compare the amplitudes seen by SUMER in low-intensity areas outside the sunspot plumes Their amplitudes vary between 5 and 15 percent, i.e., they are very similar to the amplitude seen by CDS. In only one case (Brynildsen et al. 1999a) was the amplitude higher (35 percent), approaching the minimum of 50 percent required by our case (2) to explain the oscillations seen by CDS. This suggests that our interpretation (1) is correct in most cases, i.e., there are oscillations in sunspot plumes. In fact, various combinations between the two extremes are possible, for example, the following pairs $\left(\Delta I_{\mathrm{b}} / I_{\mathrm{b}}, \Delta I_{\mathrm{p}} / I_{\mathrm{p}}\right)$ of the relative oscillation amplitudes: $(30 \%, 5 \%),(20 \%, 7.5 \%)$ or $(14 \%, 9 \%)$ for the low-intensity $I_{\mathrm{b}}$ and high-intensity $I_{\mathrm{p}}$ plasma, respectively, also satisfy the parameters chosen above. Each of these possibilities, with the exception of the extreme interpretation (2), allows oscillations in the sunspot plumes to occur.

One should also note that the relative oscillation amplitude in the CDS data is nearly constant in time, while the total intensity varies. If the background intensity $I_{\mathrm{b}}$ oscillated with amplitude $\Delta I_{\mathrm{b}}$ and the plume intensity $I_{\mathrm{p}}$ did not oscillate, the relative amplitude $\Delta I_{\mathrm{b}} /\left(I_{\mathrm{p}}+I_{\mathrm{b}}\right)$ should increase when $\left(I_{\mathrm{p}}+I_{\mathrm{b}}\right)$ decreases, and that is not observed.

Therefore, a contention that part of the sunspot plumes oscillates in the transition region emission seems consistent with the CDS observations. However, we stress that there are some areas with increased intensity which do not oscillate.

Finally, we turn to the observation of NOAA active region 8552 made with the CDS on 30 May 1999, where the oscillations have been found not only near the bright sunspot plume but also in the area of lower intensity, four times less intense than the peak of the sunspot plume, and separate from the large sunspot plume. This measurement has been possible due to a fortunate morphology of this large sunspot, where there appears to be an extension of the umbra which is not covered by the bright sunspot plume. The low-intensity feature looks as if it were magnetically rooted at one end in this umbral 
extension and plasma at transition region temperatures is seen in projection above the penumbra. The relative amplitude of the intensity oscillations in this feature is up to 15 percent, greater than the relative amplitude in the sunspot plume. We note that this area is still brighter by more than a factor of three than the average intensity above other non-oscillating parts of the penumbra and in the area surrounding the sunspot. Thus, while its brightness is not high enough to meet the intensity threshold defining a sunspot plume as proposed by Brynildsen et al. (1999b), it has two similarities to sunspot plumes: it has an enhanced brightness and appears to be connected to the umbra.

\subsection{Conclusions}

The results of this paper can be summarised as follows:

We have analysed $15 \mathrm{~s}$ cadence CDS observations made above three sunspots in the transition region lines in the temperature range $910^{4}-410^{5} \mathrm{~K}$. Intensity oscillations can be seen in all lines in this temperature range. In two cases, a chromospheric line of He I $584.3 \AA$ also shows intensity oscillations. The relative amplitude is 5-10 percent, with isolated peaks up to 15 percent. The oscillations are seen best in bright lines of O V $629.7 \AA$ and O IV $554.5 \AA$. The amplitude is largest in the O IV line $\left(1.710^{5} \mathrm{~K}\right)$ while the oscillations that reach the $410^{5} \mathrm{~K}$ plasma are significantly dampened.

The Fourier power spectrum shows significant power in the range of periods $120-210 \mathrm{~s}$ with power concentration around 160-180 s. The detailed shape of the power spectrum varies from sunspot to sunspot. A wavelet analysis shows that the oscillation period varies in different parts of the time series.

Observations made with solar tracking show a Fourier power spectrum which has most of the power concentrated near $5.8 \mathrm{mHz}(170 \mathrm{~s})$.

The wavelet analysis shows that different frequency components in the Fourier power spectrum usually correspond to different spatial locations or to frequency varying with time, and only rarely to several frequencies present simultaneously at one location.

Intensity oscillations in the CDS data are typically seen near the spatial peak of intensity corresponding to sunspot plumes. A likely interpretation, consistent with the spatial resolution of CDS, is that oscillations occur both in the sunspot plumes and in the lower intensity plasma adjacent to the plumes. In the extreme case, if the sunspot plumes did not oscillate at all, the source of oscillations would have to be in the low-intensity plasma always adjacent to sunspot plumes and the relative oscillation amplitudes in that plasma would exceed 50 percent.
Oscillations have also been found above umbra and penumbra in an area with intensity much lower than that of a typical sunspot plume but still enhanced over the chromospheric network emission.

Acknowledgements. This work was supported by the United Kingdom's PPARC. SOHO is a project of international cooperation between the European Space Agency and NASA. Wavelet software was provided by C. Torrence and G. Compo, and is available at http://paos.colorado.edu/research/wavelets/. The author thanks Drs. J. Ireland, J. Gurman and B. Fleck for helpful discussions, Drs. D. Zarro and W. Thompson for advice on SOHO analysis software, Dr. T. Kucera for advice on the SUMER instrument, and Dr. J. Saba for comments on the manuscript.

\section{References}

Bocchialini, K., \& Baudin, F. 1995, A\&A, 299, 893

Brynildsen, N., Kjeldseth-Moe, O., Maltby, P., \& Wilhelm, K. 1999a, ApJ, 517, L159

Brynildsen, N., Maltby, P., Brekke, P., Haugan, S. V. H., \& Kjeldseth-Moe, O. 1999b, Sol. Phys., 186, 141

Brynildsen, N., Maltby, P., Leifsen, T., Kjeldseth-Moe, O., \& Wilhelm, K. 2000, Sol. Phys., 191, 129

Fludra, A. 1999a, A\&A, 344, L75

Fludra, A. 1999b, Proc. 8th SOHO workshop, ESA SP-446, 327

Fludra, A., Brekke, P., Harrison, R. A., et al. 1997, Sol. Phys., 175,487

Foukal, P. V., Noyes, R. W., Reeves, E. M., et al. 1974, ApJ, 193, L143

Foukal, P. V. 1976, ApJ, 210, 575

Gurman, J. B., Leibacher, J. W., Shine, R. A., Woodgate, B. E., \& Henze, W. 1982, ApJ, 253, 939

Gurman, J. B., \& Leibacher, J. W. 1984, ApJ, 283, 859

Harrison, R. A., \& 38 co-authors 1995, Sol. Phys., 162, 233

Ireland, J., Walsh, R. W., Harrison, R. A., \& Priest, E. R. 1999, A\&A, 347, 355

Lites, B. W., White, O. R., \& Packman, D. 1982, ApJ, 253, 386

Lites, B. W. 1986, ApJ, 301, 992

Lites, B. W. 1992, in Sunspots: Theory and Observations, ed. J. H. Thomas, \& N. O. Weiss (Kluwer Academic Publishers, Dordrecht), 261

Maltby, P., Brynildsen, N., Brekke, P., et al. 1998, ApJ, 496, L117

Maltby, P., Brynildsen, N., Fredvik, T., Kjeldseth-Moe, O., \& Wilhelm, K. 1999, Sol. Phys., 190, 437

Pauluhn, A., Ruedi, I., Solanki, S. K., et al. 1999, Appl. Opt., 38,7035

Torrence, C., \& Compo, G. P. 1998, A Practical Guide to Wavelet Analysis, Bull. Amer. Meteor. Soc., 79, 61

Wilhelm, K., \& 18 co-authors 1997, Sol. Phys., 170, 75 